ARTICLE

DOI: $10.1038 / s 41467-018-04828-y$

\title{
Structural bases of TRP channel TRPV 6 allosteric modulation by 2-APB
}

Appu K. Singh ${ }^{1}$, Kei Saotome ${ }^{1}$, Luke L. McGoldrick ${ }^{1,2} \&$ Alexander I. Sobolevsky ${ }^{1}$

Transient receptor potential (TRP) channels are involved in various physiological processes, including sensory transduction. The TRP channel TRPV 6 mediates calcium uptake in epithelia and its expression is dramatically increased in numerous types of cancer. TRPV 6 inhibitors suppress tumor growth, but the molecular mechanism of inhibition remains unknown. Here, we present crystal and cryo-EM structures of human and rat TRPV6 bound to 2-aminoethoxydiphenyl borate (2-APB), a TRPV6 inhibitor and modulator of numerous TRP channels. 2-APB binds to TRPV6 in a pocket formed by the cytoplasmic half of the S1-S4 transmembrane helix bundle. Comparing human wild-type and high-affinity mutant Y467A structures, we show that 2-APB induces TRPV6 channel closure by modulating protein-lipid interactions. Mutagenesis and functional analyses suggest that the identified 2-APB binding site might be present in other members of vanilloid subfamily TRP channels. Our findings reveal a mechanism of ion channel allosteric modulation that can be exploited for therapeutic design.

\footnotetext{
${ }^{1}$ Department of Biochemistry and Molecular Biophysics, Columbia University, 650 West 168th Street, New York, NY 10032, USA. ${ }^{2}$ Integrated Program in Cellular, Molecular and Biomedical Studies, Columbia University, 650 West 168th Street, New York, NY 10032, USA. These authors contributed equally: Appu K. Singh, Kei Saotome. Correspondence and requests for materials should be addressed to A.I.S. (email: as4005@cumc.columbia.edu)
} 
$\mathrm{T}$ he transient receptor potential (TRP) ion channel superfamily comprises members that are involved in various physiological functions ranging from sensory transduction to calcium homeostasis ${ }^{1}$. Aberrant regulation of TRP channels results in various diseases ${ }^{2}$, including numerous types of cancer. TRPV6, a highly calcium-selective TRP channel, is overexpressed in endometrial cancers, leukemia, and carcinomas of the breast, prostate, colon, ovarian, and thyroid ${ }^{3-18}$. TRPV6 has been implicated in tumor development and progression, and its overexpression pattern correlates with the aggressiveness of the disease $^{4,8,9,15,19-21} \cdot \mathrm{Ca}^{2+}$ is a critical regulator of cell proliferation, suggesting a role for TRPV6 in the potentiation of calciumdependent cell proliferation and inhibition of apoptosis ${ }^{17}$. Inhibitors of TRPV6 may, therefore, offer a novel therapeutic strategy for treatment of TRPV6-rich tumors ${ }^{12,17,20,22}$. A limited number of small-molecule $e^{23-26}$ and peptide ${ }^{27}$ inhibitors of TRPV6 have been identified as potential leads for cancer treatment, but advances in drug development are hampered by lack of knowledge about the possible molecular mechanisms of TRPV6 inhibition.

A membrane-permeable compound 2-aminoethoxydiphenyl borate (2-APB), one of the few known small-molecule inhibitors of TRPV6, has been shown to attenuate tumor growth and invasiveness in human cancer cell lines in vitro ${ }^{26}$. 2 -APB was initially characterized as an inhibitor of $\operatorname{Ins}(1,4,5) \mathrm{P} 3$ receptor-induced $\mathrm{Ca}^{2+}$ release ${ }^{28}$, but was later shown to modulate the functions of different ion channels, including calcium release-activated ${ }^{29}$ and two-pore potassium ${ }^{30}$ channels. 2-APB modulation of TRP channels ${ }^{31}$, includes activation of TRPV1, TRPV2, TRPV3, TRPA1, and TRPM $6^{32-35}$ and inhibition of TRPM2, TRPM7, TRPC3, TRPC6, and TRPC $7^{36-38}$. The promiscuousness of 2-APB makes it an important research tool to characterize physiological function and biophysical properties of ion channels. However, the potential of 2-APB as a lead compound for drug design remains limited because its mechanisms of action remain poorly understood. To address this knowledge gap, we embarked on structural studies of TRPV6 inhibition by 2 -APB.
Here, we solve cryo-EM and crystal structures of human and rat TRPV6 in complex with 2-APB, which binds in a pocket formed by the cytoplasmic half of the S1-S4 transmembrane helix bundle. By comparing our structures, we find that 2-APB induces TRPV6 channel closure by modulating protein-lipid interactions. Mutagenesis and functional analyses suggest that the 2-APB binding site is likely present in other members of the vanilloid subfamily of TRP channels. Our proposed mechanism of TRPV6 inhibition by 2 -APB, therefore, contributes to the general principles of TRP channel regulation by small hydrophobic molecules.

\section{Results}

Crystal structure of rat TRPV6 in complex with 2-APB. We used the TRPV6 ${ }^{*}$ construct that we developed earlier by modifying rat TRPV6 (rTRPV6) for crystallization (see Methods). TRPV6 ${ }^{*}$ exhibits $\mathrm{Ca}^{2+}$ permeability and $\mathrm{Gd}^{3+}$ block similar to the wild-type channel ${ }^{39}$. Importantly, in fluorimetric assays 2 -APB inhibited calcium uptake through TRPV6* (Fig. 1a, b) with a similar efficacy $\left(\mathrm{IC}_{50}=156 \pm 20 \mu \mathrm{M}, n=3\right.$ ) as in wild-type channels $\left(\mathrm{IC}_{50}=184 \pm 8 \mu \mathrm{M}, n=4\right)$. We solved the crystal structure of TRPV6 ${ }^{*}$ in complex with 2 -APB $\left(\mathrm{TRPV}^{*}{ }^{*}\right.$ $\mathrm{APB}$ ) by molecular replacement using the ligand-free TRPV $6^{*}$ structure $^{39}$ as a search model (Supplementary Table 1). The TRPV6 $^{*}{ }_{2-A P B}$ structure (Fig. 2a, b) does not exhibit substantial conformational rearrangements when compared to TRPV6* (the root mean square deviation, RMSD $=0.505$ ). TRPV6 has four subunits that form a transmembrane domain (TMD) with a central ion channel pore, an intracellular skirt domain enclosing a large cavity, and amphipathic TRP helices that run nearly parallel to the membrane and interact with both the TMD and the skirt. The TMD is composed of transmembrane helices S1-S6 and a re-entrant pore loop (P-loop) between $\mathrm{S} 5$ and S6. The first four transmembrane helices form a bundle to constitute the S1-S4 domain. The domain homologous to the S1-S4 domain acts as the voltage sensor in voltage-gated channels ${ }^{40}$, but its role in allosteric gating of TRP channels remains obscure. The pore domain of each subunit includes,
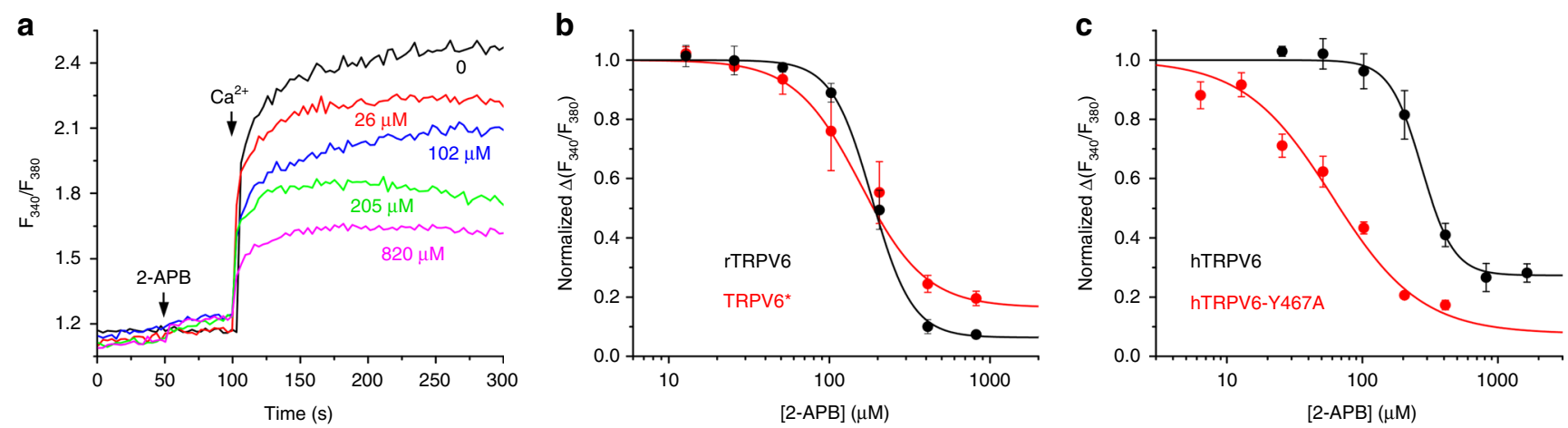

Fig. $12-A P B$ inhibition of $\mathrm{Ca}^{2+}$ uptake through wild-type and mutant TRPV6 channels. a Ratiometric Fura-2 fluorescence curves recorded from HEK 293 cells expressing TRPV6 $6^{*}$ in response to application of $2 \mathrm{mM} \mathrm{Ca}^{2+}$ after preincubation of cells in different concentrations of 2-APB. Arrows indicate the times when 2-APB and $\mathrm{Ca}^{2+}$ were added. These experiments were repeated independently three times with similar results. b Dose-response curves for 2-APB inhibition of $\mathrm{Ca}^{2+}$ uptake calculated for wild-type rTRPV6 (black) and TRPV6* (red) ( $n=3$ for all measurements). The changes in the fluorescence intensity ratio at 340 and $380 \mathrm{~nm}\left(F_{340} / F_{380}\right)$ evoked by addition of $2 \mathrm{mM} \mathrm{Ca}^{2+}$ after preincubation with various concentrations of 2-APB were normalized to the maximal change in $F_{340} / F_{380}$ after addition of $2 \mathrm{mM} \mathrm{Ca}^{2+}$ in the absence of 2-APB. Straight lines through the data points are fits with the logistic equation, with the mean \pm SEM values of the half-maximum inhibitory concentration $\left(I_{50}\right), 184 \pm 8$ and $156 \pm 20 \mu \mathrm{M}$, and the maximal inhibition, $93.6 \pm 1.1 \%$ and $83.5 \pm 2.9 \%$, for rTRPV6 and TRPV6 ${ }^{\star}$, respectively. c Dose-response curves for 2 -APB inhibition of Ca ${ }^{2+}$ uptake calculated for wild-type hTRPV6 (black) and hTRPV6-Y467A (red) ( $n=3$ for all measurements) are fits with the logistic equation, with the parameter values IC $50=274 \pm 27$ and $60 \pm 13 \mu \mathrm{M}$, and the maximal inhibition, $72.6 \pm 2.7 \%$ and $92.4 \pm 8.1 \%$, for hTRPV6 and hTRPV6-Y467A, respectively. The leftward shift of the 2-APB dose-response curve of hTRPV6-Y467A, when compared to the dose-response curve of wild-type hTRPV6, indicates an increased affinity of the channel for 2-APB. Error bars represent SEMs 

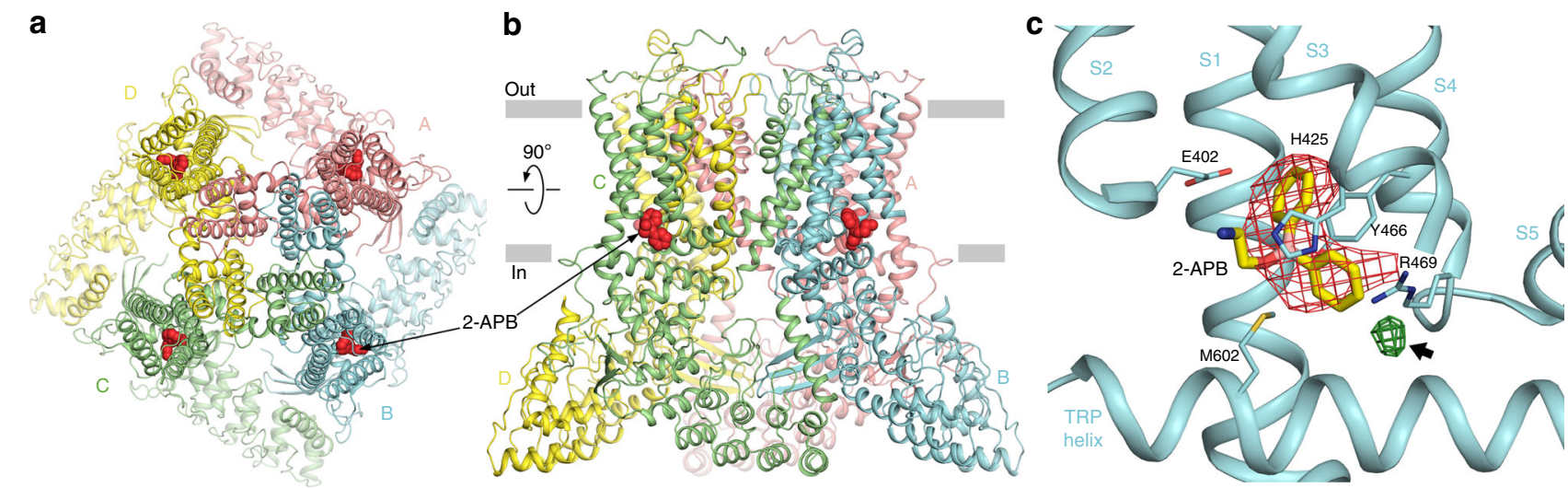

Fig. 2 Crystal structure of rat TRPV6 in complex with 2-APB. a, b Top (a) and side (b) views of TRPV6* in complex with 2-APB. The molecules of 2-APB are shown as red space-filling models. Four TRPV6* subunits are colored pink, cyan, green, and yellow. c Close-up view of the 2-APB binding site with $2-\mathrm{APB}$ (yellow) and surrounding residues shown as sticks. Red mesh shows positive electron density for $2-\mathrm{APB}$ in the Fo-Fc omit map contoured at $3 \sigma$. Green mesh indicated by the arrow shows electron density for the brominated derivative of 2-APB (2-APB-Br) in the anomalous difference Fourier map contoured at $3 \sigma$

S5, the P-loop, and S6, and is packed against the S1-S4 domain of the neighboring subunit in a domain-swapped arrangement ${ }^{39,41}$.

2-APB binding pocket in rat TRPV6. In each subunit of the TRPV $^{*}{ }_{2-\mathrm{APB}}$ tetramer, a pocket formed by the intracellular portions of the S1-S4 helices and the membrane-facing sides of the amphipathic TRP helices contained a strong density that matches the size and shape of 2-APB and was not observed in the TRPV6 ${ }^{\star}$ structure (Fig. 2c, Supplementary Figure 1a, b). To verify this $2-\mathrm{APB}$ binding site crystallographically, we used a heavy atom bromine derivative of 2-APB (2-APB-Br; Fig. 3a, b) and collected diffraction data at a wavelength of $0.92 \AA$ from crystals of TRPV6 ${ }^{*}$ grown in the presence of $2-\mathrm{APB}-\mathrm{Br}$ (Supplementary Table 1). These data revealed an anomalous difference $\mathrm{Br}$ peak at the putative 2-APB electron density and unambiguously identified the orientation of 2-APB in the binding pocket (Fig. 2c, Fig. 3). Notably, this binding pocket is distinct from the pocket at the S4-S5 interface that binds an activating lipid in TRPV6 ${ }^{41}$, agonists, such as resiniferatoxin (RTX) and capsaicin, and antagonists, such as capsazepine (CPZ), in TRPV $1^{42}$, and the inhibitor econazole in TRPV $5^{43}$. However, the 2-APB binding site in TRPV ${ }^{*}$ overlaps with a lipid binding site found in TRPV $1^{42}$, TRPV $2^{44,45}$, TRPV $5^{43}$, and TRPV6 ${ }^{41}$ (Supplementary Figure $1 \mathrm{c}-\mathrm{f})$; a $\mathrm{Ca}^{2+}$ binding site in TRPM4 ${ }^{46}$; putative binding sites for phosphoinositides in TRPA1 ${ }^{47}$; and menthol in TRPM8 ${ }^{48}$, suggesting an important role for this site in modulating TRP channel function.

2 -APB binding is apparently mediated by multiple interactions (Fig. 2c). E402 in S2 appears to interact with the amino group of the 2-APB tail. In addition, the two phenyl rings of 2-APB are surrounded by the imidazole group of $\mathrm{H} 425$ in S3, the guanidinium group of R469 in S4, the hydrophobic side chains of Y466 in the S4-S5 linker, and M602 in the TRP domain.

Binding Site Mutations Increase 2-APB Modulation Potency. To further characterize the 2-APB binding site and to examine the contribution of individual residues to 2-APB inhibition, we introduced alanine substitutions at residues in the 2-APB binding pocket (E402, H425, Y466, R469, and M602) and tested 2-APB inhibition. In stark contrast to our expectations, the mutations did not weaken the inhibitory potency of 2-APB, but either had little effect $\left(\mathrm{IC}_{50}=165 \pm 32 \mu \mathrm{M}, n=3\right.$ for $\mathrm{H} 425 \mathrm{~A} ; \mathrm{IC}_{50}=98 \pm 19 \mu \mathrm{M}$, $n=3$ for M602A), or resulted in a remarkable 8- to 50 -fold increase in 2-APB potency $\left(\mathrm{IC}_{50}=7.5 \pm 2.1 \mu \mathrm{M}, n=3\right.$ for $\mathrm{E} 402 \mathrm{~A} ; \mathrm{IC}_{50}=$ $3.62 \pm 0.54 \mu \mathrm{M}, n=4$ for $\mathrm{Y} 466 \mathrm{~A} ; \mathrm{IC}_{50}=23.4 \pm 1.6 \mu \mathrm{M}, n=4$ for R469A) compared to wild-type channels (Fig. 4a).

The strongest increase in potency of calcium uptake inhibition by 2 -APB was observed for the mutant TRPV6 ${ }^{*}-Y_{466 A}$. To shed light on the structural basis of this phenotype, we crystallized TRPV $^{*}-$ Y466A in the presence and absence of 2-APB (Fig. 4b, Supplementary Table 1 ). In the presence of $2-A P B$, a strong density with the characteristic shape of $2-\mathrm{APB}$ is present in the $\mathrm{S} 1-\mathrm{S} 4$ binding pocket, similar to $\mathrm{TRPV}^{*}{ }_{2-\mathrm{APB}}$. Interestingly, both the shape of the 2-APB density in the Fo-Fc map of

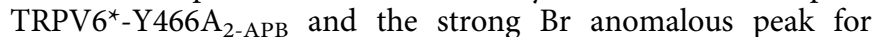
TRPV6 $^{*}$-Y466A co-crystallized with 2-APB-Br (Fig. 3) indicate that the binding pose for $2-\mathrm{APB}$ in TRPV6*-Y466 $\mathrm{A}_{2-\mathrm{APB}}$ is

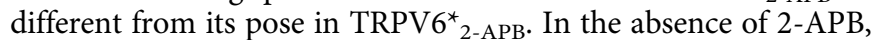
the C-terminal (cytoplasmic) part of S2 in TRPV6*-Y466A is splayed away from the binding pocket by $\sim 4 \AA$ relative to its position in TRPV6*-Y466A ${ }_{2-A P B}$ (Fig. 4b). In turn, 2-APB seems to glue the $S 2$ helix back, perhaps by virtue of its tail amino group interaction with E402 (Fig. 4c).

Y466 in TRPV6* is highly conserved throughout the entire TRPV subfamily (Supplementary Figure 2). We wondered whether mutating the homologous tyrosine in TRPV1, TRPV2, and TRPV 3 would affect the potency of $2-A P B$ as an agonist ${ }^{32}$ for these channels. Indeed, for all three, we observed leftward shifts of their 2-APB concentration dependencies (Fig. 5a-c), indicating $3-$, 40-, and 20-fold increases in 2-APB agonist potency for TRPV1, TRPV2, and TRPV3, respectively. In available structures of TRPV $1^{42}$ and TRPV $2^{44,45}$, the tyrosine side chain projects into the 2-APB binding pocket in a fashion similar to Y466 in TRPV6* (Fig. 5d, e), suggesting its conserved structural role in 2-APBdependent modulation. On the other hand, the lack of measurable 2-APB modulation of TRPV4 and TRPV $5^{32}$ despite high sequence conservation (Supplementary Figure 2), suggests that there are other determinants of modulation of TRPV channels by 2 -APB.

The increased potency of 2-APB modulation observed in inhibited (TRPV6) and activated (TRPV1-3) channels caused by the conserved tyrosine substitution in S4 supports the idea that these channels share a common site for 2-APB binding. We hypothesize that the increase, rather than reduction of $2-\mathrm{APB}$ potency caused by mutations in its binding pocket is rooted in competition between 2-APB and the S1-S4 lipid (lipid 1, see the 
a<smiles>NCCOB(c1ccccc1)c1ccccc1</smiles>

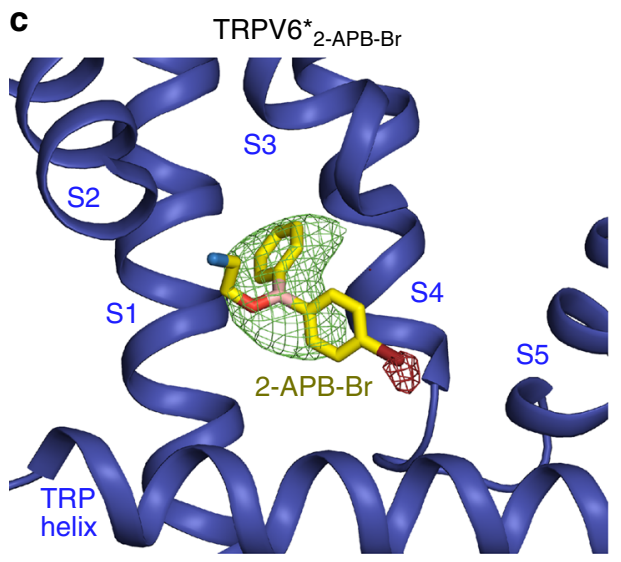

e

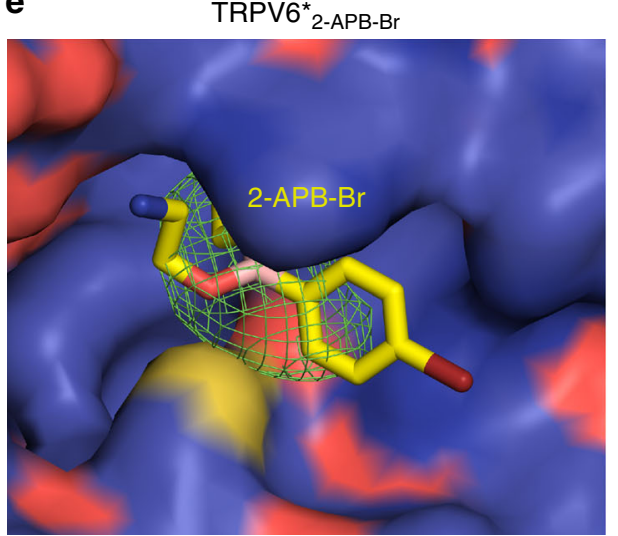

b<smiles>NCCOB(c1ccccc1)c1ccc(Br)cc1</smiles>

d

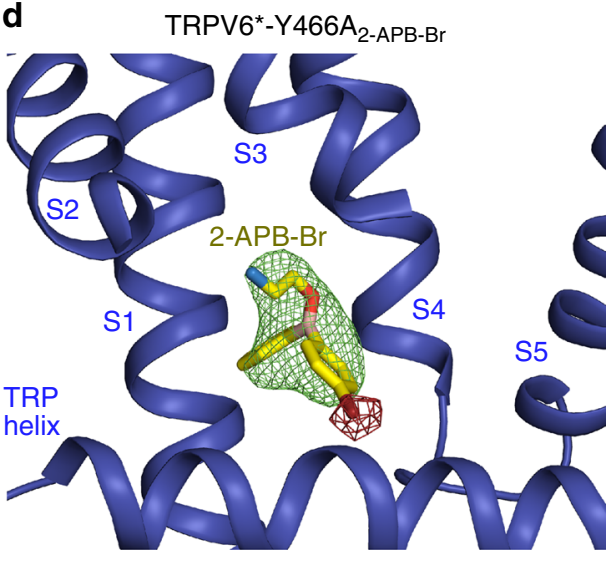

f

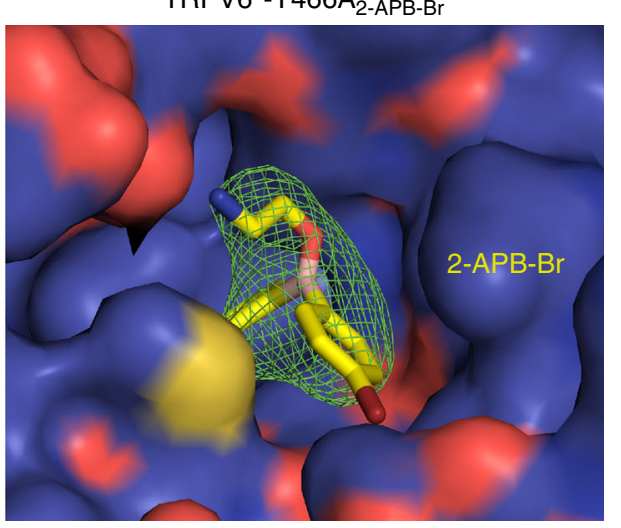

Fig. 3 Binding of brominated 2-APB to TRPV6* and TRPV6*-Y466A. a, b Chemical structures of 2-APB (a) and 2-APB-Br (b). c, d Close-up view of the

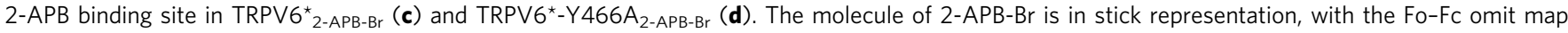
$(2.5 \sigma)$ shown as a green mesh and the anomalous difference Fourier maps at $3 \sigma$ for TRPV6 ${ }^{\star}{ }_{2-\mathrm{APB}-\mathrm{Br}}$ and $5.5 \sigma$ for TRPV $6^{\star}-\mathrm{Y} 466 \mathrm{~A}_{2-\mathrm{APB}-\mathrm{Br}}$ shown as a brown mesh. e, $\mathbf{f}$ Surface representation of the 2-APB binding pocket in TRPV6 ${ }^{\star}$ 2-APB-Br $(\mathbf{e})$ and TRPV6*-Y $466 \mathrm{~A}_{2-\mathrm{APB}-\mathrm{Br}}$ (f). Note the different orientations (poses) of the 2-APB-Br molecule in the two structures
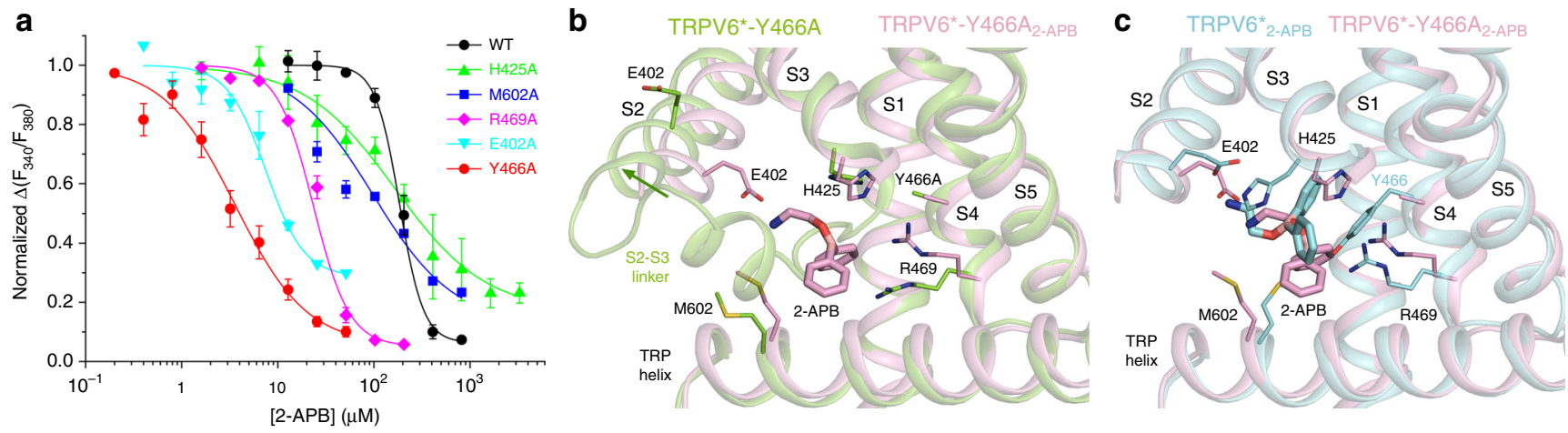

Fig. 4 Probing of 2-APB binding site in TRPV6 by mutagenesis. a Dose-response curves for 2-APB inhibition of wild-type and mutant rat TRPV6 channels with residues in the 2-APB binding pocket individually substituted to alanine. Error bars represent SEMs $(n=3-7)$. $\mathbf{b}$ Close-up view of superposition of the 2-APB binding pocket in the TRPV6*-Y466A (green) and TRPV6*-Y466A ${ }_{2-A P B}$ (pink) crystal structures with the 2-APB molecule and surrounding residues shown as sticks. Green arrow indicates displacement of $\mathrm{S} 2$ in the absence of 2-APB, accompanied by increased order in the S2-S3 linker. c Close-up view of superposition of the 2-APB binding pocket in the TRPV6 ${ }_{2-A P B}$ (cyan) and TRPV6 ${ }^{*}-Y_{466 A_{2-A P B}}$ (pink) crystal structures 

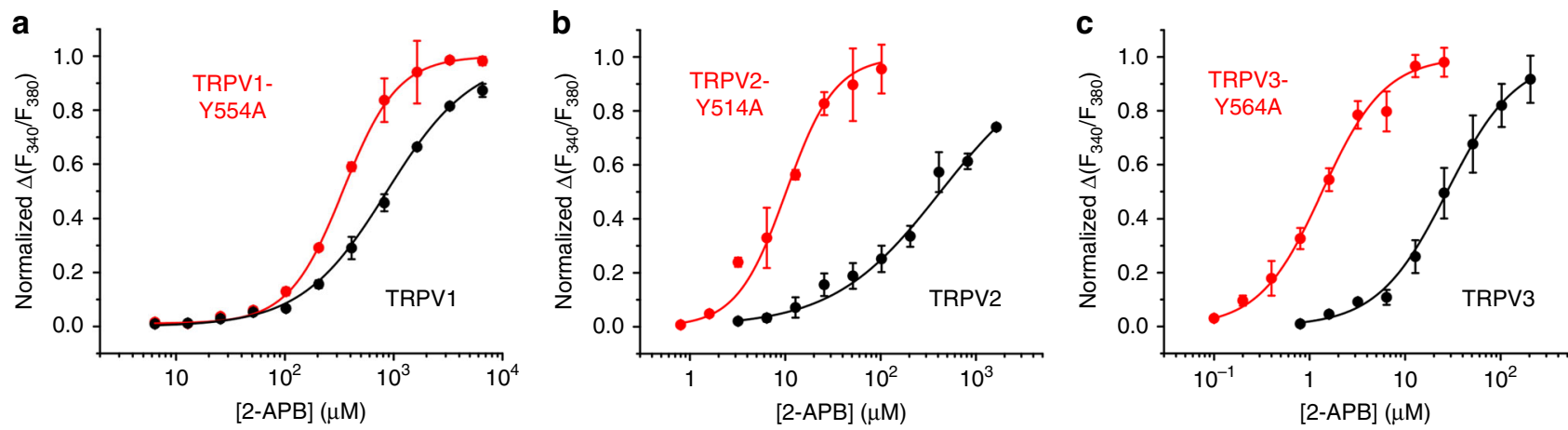

d

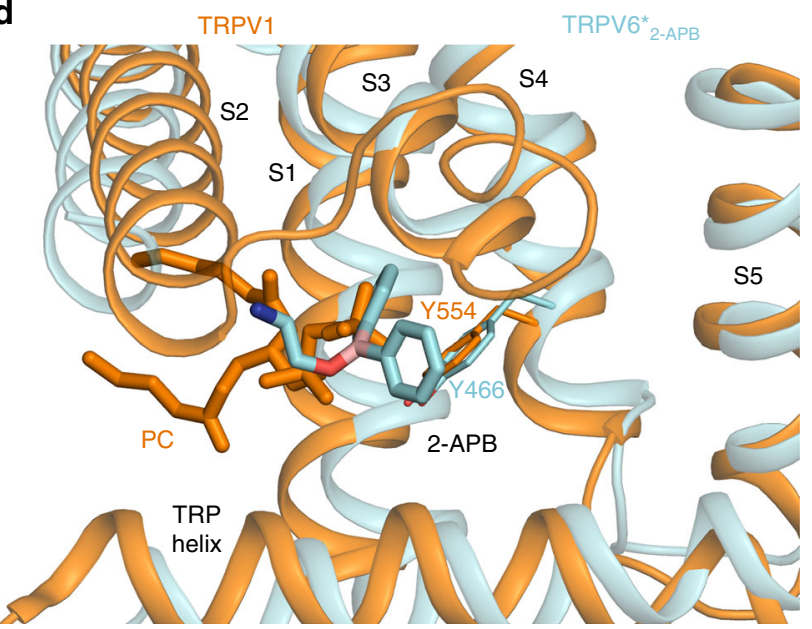

e TRPV2

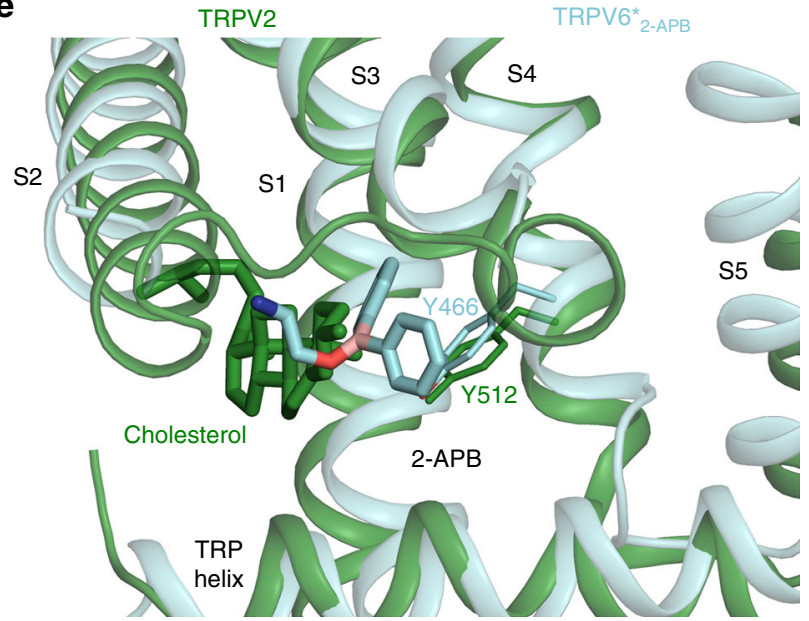

Fig. 5 Probing of the 2-APB binding site in different TRPV channels. a-c Dose-response curves for 2-APB activation of wild-type (black) and mutant (red) rat TRPV1 (a), rat TRPV2 (b), and mouse TRPV3 (c) channels with alanine substitution of the conserved tyrosine in the presumed 2-APB binding pocket. Each data point represents an average of $n=3-7$ measurements with error bars representing SEMs. d, e Close-up view of superposition of the 2-APB binding pocket in the TRPV6 ${ }_{2-A P B}$ crystal structure (cyan) and the corresponding regions of rat TRPV1 (d) PDB ID: 5IRX, orange and rabbit TRPV2 (e) PDB ID: 5AN8, green. Phosphatidylcholine (PC), cholesterol and 2-APB molecules as well as the side chain of the conserved tyrosine are shown as sticks

next section) that occupies this site in the absence of 2 -APB (Fig. 5d, e, Supplementary Figure 1). Therefore, the lack of 2-APB modulation of TRPV4 and TRPV5 ${ }^{32}$ might be due to tighter binding of the S1-S4 lipid and inability of 2-APB to displace it. In turn, a more loose binding pocket, such as the one observed in TRPV6*-Y466A (Fig. 4b), might cause reduction in TRPV6* affinity to the S1-S4 lipid and, as a result, increased affinity to 2 $\mathrm{APB}$. We decided to further explore the structural mechanism of 2 -APB inhibition by comparing TRPV6 structures in the open and 2-APB-inhibited closed states.

2-APB-Induced Closure of Human TRPV6. We have only been able to crystallize rat TRPV6 in the closed state, precluding a clear view of conformational changes associated with 2-APBdependent inhibition. We thus turned to human TRPV6 (hTRPV6), of which we recently resolved the open-state structure by cryo-electron microscopy (cryo-EM) ${ }^{41}$. Unfortunately, our attempts to determine the structure of wild-type hTRPV6 in complex with 2 -APB were not successful, likely due to lowaffinity binding $\left(\mathrm{IC}_{50}=274 \pm 27 \mu \mathrm{M}, n=3\right.$, Fig. $\left.1 \mathrm{c}\right)$. In order to increase the affinity of hTRPV6 to 2-APB, we introduced the Y467A mutation, homologous to Y466A in TRPV6*. As expected, hTRPV6-Y467A showed 4.5 -fold increased potency of 2-APB inhibition $\left(\mathrm{IC}_{50}=60 \pm 13 \mu \mathrm{M}, n=3\right)$ compared to hTRPV6 (Fig. 1c). We determined the cryo-EM structure of apo hTRPV6-Y467A (Supplementary Table 2), which is in the open-state conformation (Fig. 6a, c, e and Supplementary Figure $3 \mathrm{a}, \mathrm{c}, \mathrm{e}, \mathrm{g})$, and it is nearly identical $(\mathrm{RMSD}=0.765)$ to the cryo-EM structure of wild-type hTRPV6 (Fig. 7). Lipid$s$ are observed in positions similar to those seen in hTRPV6 ${ }^{41}$; lipid 1 is bound in a pocket formed by the intracellular half of the S1-S4 domains and C-terminal part of TRP helix, while lipid 2 is above the S4-S5 linker and contacts both S3 and S4 of the same subunit, and S5-S6 of the neighboring subunit. We then solved the cryo-EM structure of hTRPV6-Y467A in complex with 2-APB (Fig. 6b, d, f, Supplementary Figure 3b, d, f, h, Supplementary Table 2). Clear density for 2-APB is present in each subunit of hTRPV6-Y467 $\mathrm{A}_{2-\mathrm{APB}}$ tetramer (Fig. 6f), at locations very similar to the binding sites in TRPV6*-Y466 $\mathrm{A}_{2-\mathrm{APB}}$ (Fig. 4b).

Most importantly, a pore radius calculation confirmed that channel of hTRPV6-Y467 $\mathrm{A}_{2-\mathrm{APB}}$ is in the closed conformation (Fig. 8), allowing direct structural comparison with the highresolution open-state structure of hTRPV6 (Fig. 8d). 2-APB binding is accompanied by movement of F425 and P424 in S3 toward the S4-S5 linker. As a result, hydrophobic residues from the S2-S3 linker (I420), N-terminal portion of S3 (P424, F425, V427, and L428), C-terminal portion of S4 (M466), and S4-S5 linker (I486 and F487) come closer together to form a hydrophobic cluster at the bottom of the binding site for the activating lipid (lipid 2). In both hTRPV6 ${ }^{41}$ and hTRPV6$\mathrm{Y} 467 \mathrm{~A}$, the activating lipid head penetrates this area to interact with Q483 and R470 (Fig. 9a, b). In the hTRPV6-Y467 $\mathrm{A}_{2-\mathrm{APB}}$ structure, the activating lipid head is extruded by the hydrophobic cluster, and is correspondingly shifted extracellularly. This activating lipid movement is similar to that observed in 
a

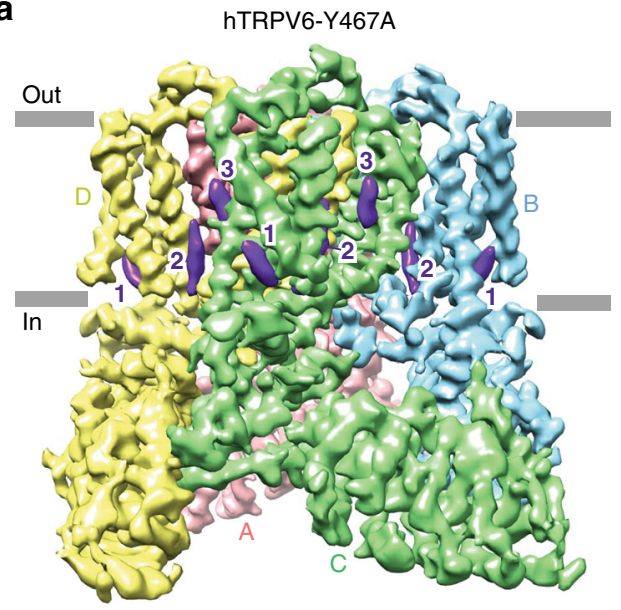

C

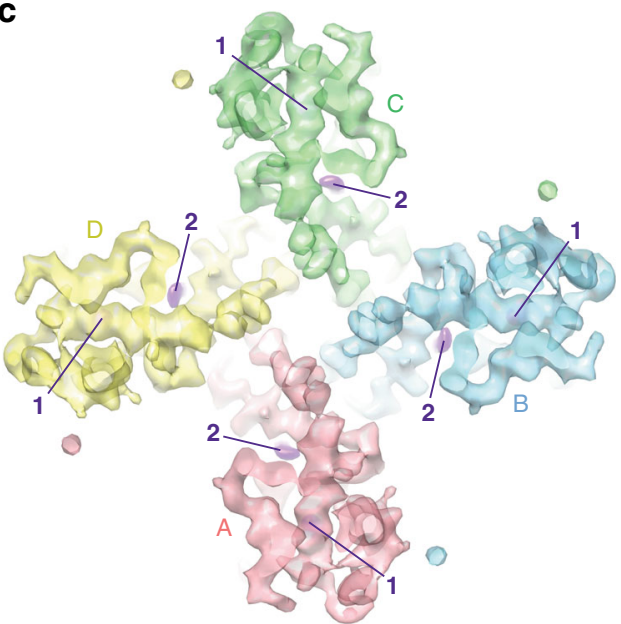

e

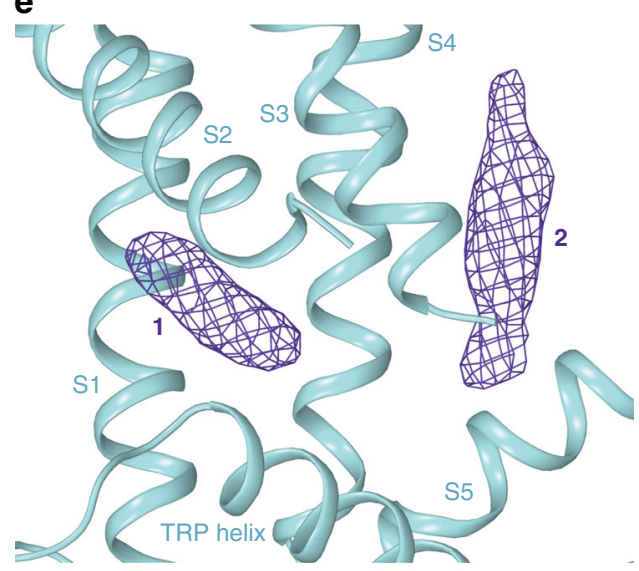

b

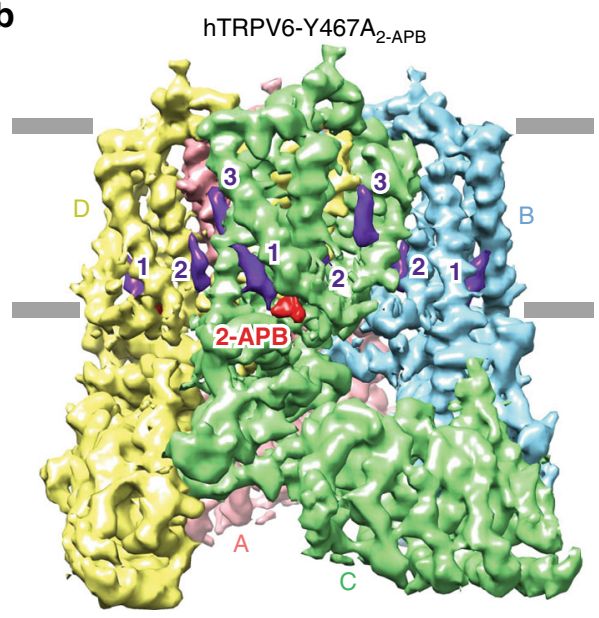

d

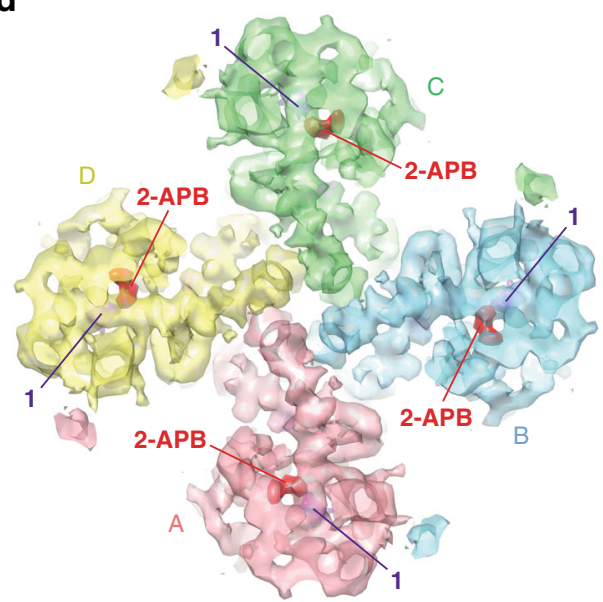

f

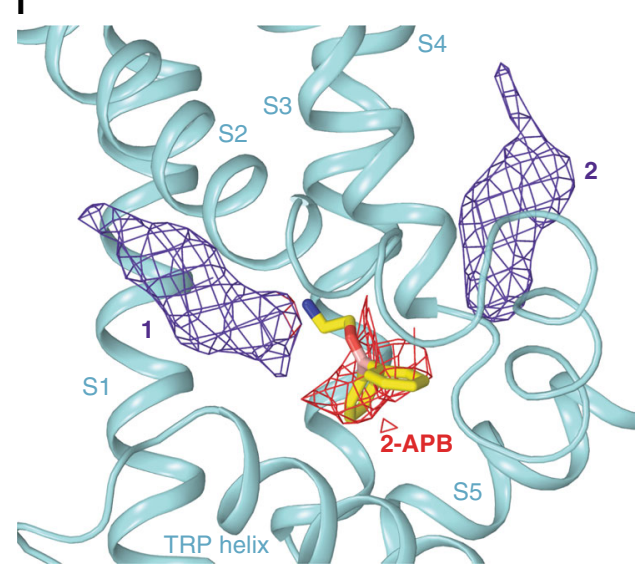

Fig. 6 Open and 2-APB-bound closed cryo-EM structures of human TRPV6. a, b 3D reconstructions of hTRPV6-Y467A (a) and hTRPV6-Y467A 2 -APB (b) viewed parallel to the membrane, with cryo-EM density for hTRPV6 subunits colored pink, cyan, green, and yellow; lipid in purple and 2-APB in red. c, d Semi-transparent cryo-EM density for the ion channel in open hTRPV6-Y467A (c) and closed hTRPV6-Y467A 2 -APB (d) viewed intracellularly. e, $\mathbf{f}$ Close-up views of the binding pocket of 2-APB (yellow) in hTRPV6-Y467A (e) and hTRPV6-Y467A 2 -APB (f). Red and purple mesh shows cryo-EM density for 2-APB and lipids, respectively

hTRPV6-R470E ${ }^{41}$, where the R470E mutation switched hTRPV6 from the open to closed state (Fig. 9c, d).

\section{Discussion}

Comparing the open-state structures of hTRPV6 and the 2-APB-bound closed-state structures, we conclude that 2-APB allosterically modulates TRPV6 channel conformation through its perturbation of lipid-protein interactions (Fig. 10, Supplementary Movie 1). Competing for the same binding site (e.g., see Supplementary Figure 1f), 2-APB wrings out the S1-S4 lipid. This promotes the formation of a hydrophobic cluster that squeezes out the activating lipid relative to its binding 
a

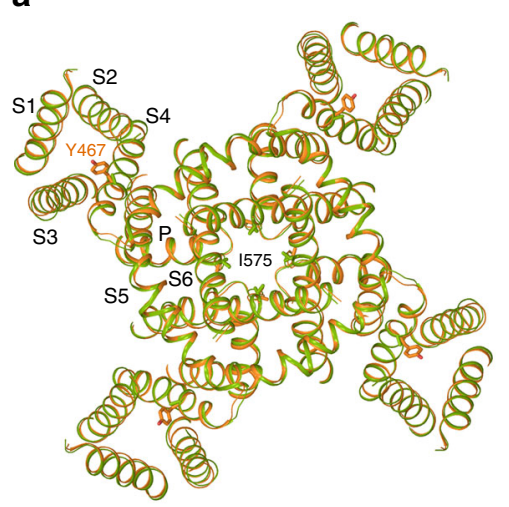

b

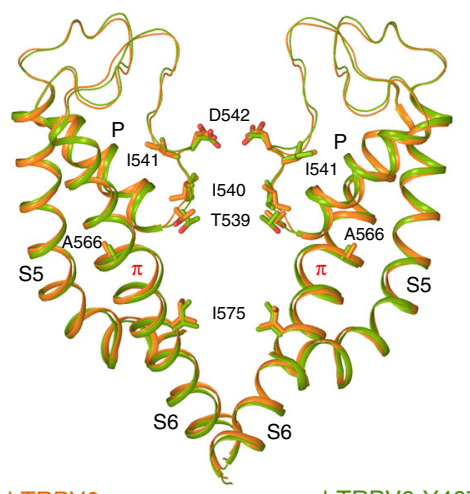

C

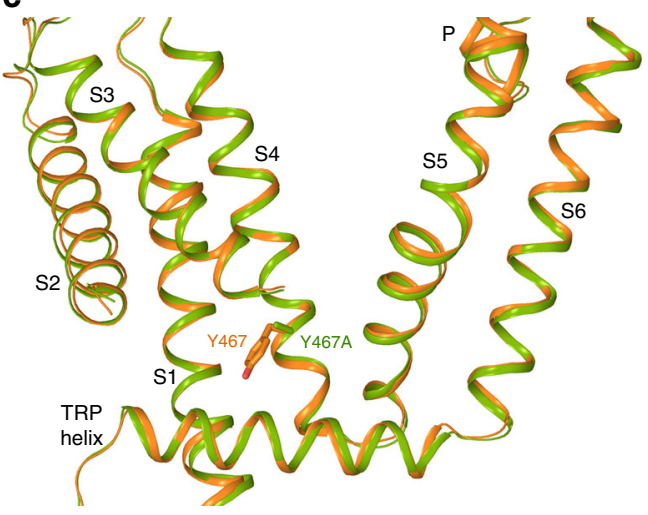

Fig. 7 Comparison of hTRPV6 and hTRPV6-Y467A cryo-EM structures. a-c Superimposed are the transmembrane domain viewed intracellularly (a) and the pore-forming region with the front and back subunits omitted for clarity (b), and the transmembrane domain of a single subunit (c) viewed parallel to the membrane for the cryo-EM structures of hTRPV6 (orange, PDB ID: 6BO8) and hTRPV6-Y467A (green). Residues contributing to the selectivity filter and gating as well as Y467 and Y467A are shown as sticks. The local $\pi$ helical conformations of S6 associated with the open channel are indicated (red labels)
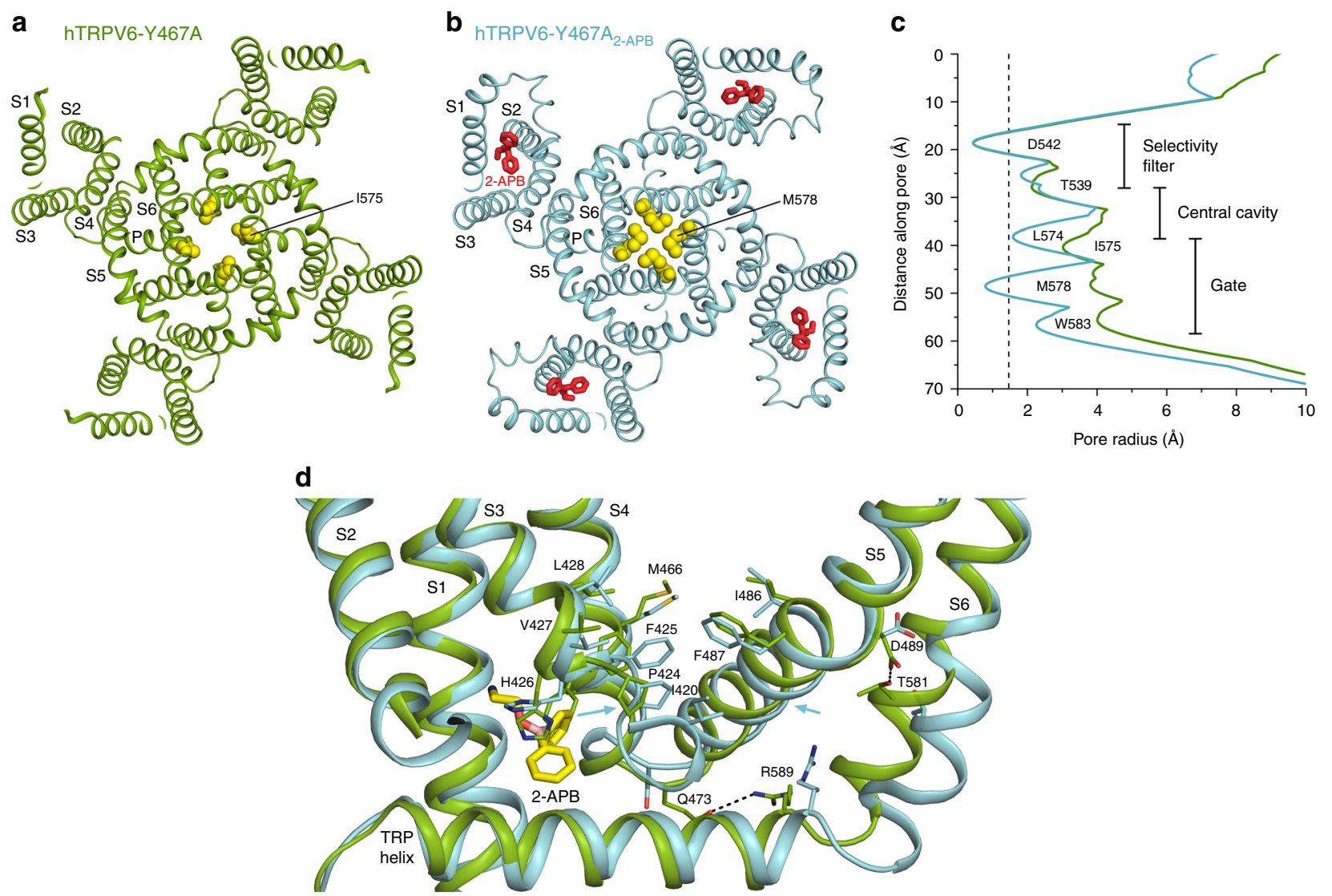

Fig. 8 Open and closed pores in cryo-EM structures of hTRPV6-Y467A and hTRPV6-Y467A 2 -APB and 2-APB-induced conformational changes.

a, b Intracellular view of the transmembrane domain in open-pore hTRPV6-Y467A (a) and closed-pore hTRPV6-Y467A 2 -APB (b). Residues forming the narrowest part of the pore in the gate region, 1575 in hTRPV6-Y467A (a) and M578 in hTRPV6-Y467A 2-APB (b), are shown as space-filling models (yellow). Molecules of 2-APB are shown as sticks (red). c Pore radius calculated using HOLE ${ }^{66}$ for hTRPV6-Y467A (green) and hTRPV6-Y467A 2 -APB (cyan). d Superposition of hTRPV6-Y467A (green) and hTRPV6-Y467A 2 -APB (cyan), with hydrophobic residues, residues involved in 2-APB binding, residues important for gating, and 2-APB shown as sticks. Blue arrows illustrate movement of the hydrophobic residues to form the hydrophobic cluster. Dashed lines illustrate hydrogen bonds that stabilize the open state

locus in the open state structure of TRPV6 ${ }^{41}$. The accompanying rearrangements of the transmembrane helices eliminate hydrogen bonds between Q473 in the S4-S5 linker and R589 in the TRP helix, and between D489 in the S5 helix and T581 in the S6 helix. These bonds compensate for the unfavorable $\alpha$-to- $\pi$ helical transition in S6 that occurs during channel opening and their removal makes S6 $\alpha$-helical and promotes channel closure. 
a

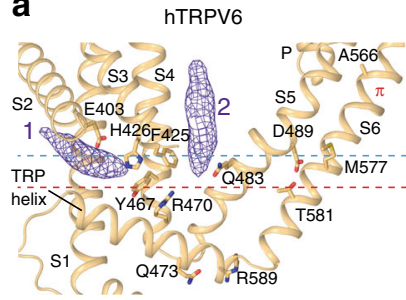

b

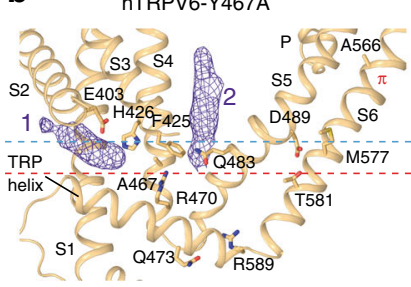

C $\quad$ TTRPV6-Y467A $2-A P B$

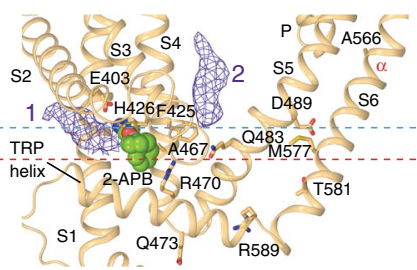

d hTRPV6-R470E

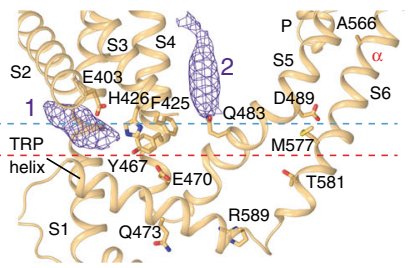

Fig. 9 Densities for S1-S4 and activating lipids in cryo-EM structures of hTRPV6. a-d Close-up views of the transmembrane domain of a single subunit in the cryo-EM structures of hTRPV6 (a PDB ID: 6BO8), hTRPV6-Y467A (b), hTRPV6-Y467A 2-APB (c), and hTRPV6-R470E (d PDB ID: 6BOA) viewed parallel to the membrane. The molecule of 2-APB is shown as a space-filling model (green). Purple mesh shows cryo-EM density for the S1-S4 (1) and activating (2) lipids, low pass filtered to the same ( $4.44 \AA$ ) resolution. Red and blue dashed lines indicate the lowest (most intracellular) levels reached by the activating lipids in the open (hTRPV6 and hTRPV6-Y467A) and closed (hTRPV6-Y467A 2-APB and hTRPV6-R470E) structures, respectively. The local $\pi$ and $\alpha$ helical conformations of $\mathrm{S} 6$ associated with the open and closed states, respectively, are indicated (red labels)

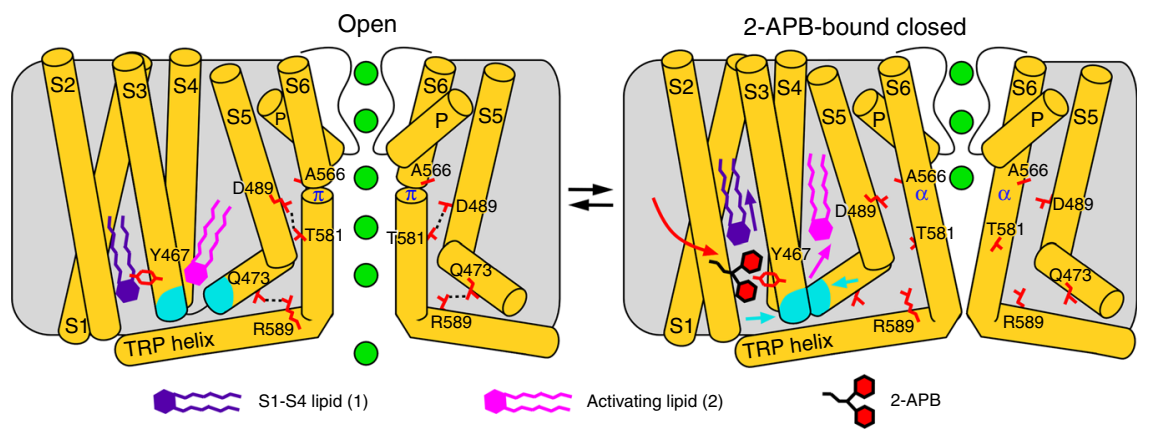

Fig. 10 Mechanism of TRPV6 inhibition by 2-APB. Cartoons represent the structural changes that start with binding (red arrow) of 2-APB, which displaces the S1-S4 lipid (lipid 1, purple) and promotes formation of the hydrophobic cluster (cyan). Formation of the cluster displaces the activating lipid (lipid 2, pink) and eliminates hydrogen bonds (dashed lines), which stabilize the open state by energetically compensating the unfavorable $\alpha$-to- $\pi$ helical transition in S6. As S6 turns $\alpha$-helical, the channel closes and its pore becomes impermeable to ions (green spheres)

We hypothesize that 2-APB similarly modulates the activity of other TRPV channels by manipulating their interactions with bound lipids, but that the functional outcome (activation, inhibition, or no effect) depends on the exact nature of the channel-lipid complex and whether the lipids have a positive or negative regulatory role in channel gating. The 2-APB binding site identified in the present study can, therefore, be exploited for the design of small hydrophobic molecules that can alter channel function in a specific way, possibly leading to novel therapeutic strategies.

\section{Methods}

Construct. For crystallization experiments, we used the previously developed construct TRPV6 ${ }^{* 39}$, which includes wild-type rat TRPV6 (rTRPV6) residues 1-668 and three point mutations (I62Y, L92N, and M96Q), and the TRPV6 ${ }^{*}-$ Y466A construct, which contains an additional point mutation, Y466A. For calcium uptake measurement experiments, we used TRPV6*, rTRPV6, and rTRPV6 with individual alanine substitutions (rTRPV6-E402A, rTRPV6H425A, rTRPV6-Y466A, rTRPV6-R469A, and rTRPV6-M602A), wild-type human TRPV6 (hTRPV6), hTRPV6 with a single tyrosine-to-alanine substitution (hTRPV6-Y467A), wild-type rat TRPV1 (rTRPV1), rat TRPV2 (rTRPV2) and mouse TRPV3 (mTRPV3), and their mutant versions with single tyrosine-toalanine substitutions (rTRPV1-Y554A, rTRPV2-Y514A, and mTRPV3-Y564A, respectively). For cryo-EM experiments, we used hTRPV6 and hTRPV6-Y467A constructs.

Expression and Purification. The TRPV6 $6^{*}$, TRPV6 ${ }^{*}$-Y466A, and hTRPV6-Y467A constructs were expressed and purified as previously described for TRPV6 ${ }_{\text {cryst }}{ }_{4}$ and hTRPV $6^{41}$ with slight modification. Briefly, for our crystallographic studies, TRPV6 ${ }^{*}$ was introduced into a pEG BacMam vector ${ }^{50}$ with a C-terminal thrombin cleavage site (LVPRG) followed by eGFP and a streptavidin affinity tag (WSHPQFEK) for expression in baculovirus-transduced, suspension-adapted HEK $293 \mathrm{GnTI}^{-}$cells (ATCC, Cat\#CRL-3022). For our cryo-EM studies, hTRPV6-Y467A was introduced into a similar pEG BacMam vector, but without GFP. In total, $48-72 \mathrm{~h}$ after transduction with baculovirus virus, HEK $293 \mathrm{GnTI}^{-}$cells were harvested, washed with PBS pH 8.0, and after sonication, cellular membranes were prepared. The membranes were then mechanically homogenized, and solubilized for $1 \mathrm{~h}$ in $150 \mathrm{mM} \mathrm{NaCl}, 20 \mathrm{mM}$ Tris-HCl pH 8.0, 1\% DDM (n-dodecyl- $\beta$-D-maltopyranoside), $0.1 \%$ cholesteryl hemisuccinate (CHS), and $1 \mathrm{mM} \beta$-mercaptoethanol (BME) for hTRPV6-Y467A. CHS was omitted while purifying TRPV6 $6^{\star}$ and TRPV6*-Y466A. After solubilizing the membranes, any remaining insoluble material was pelleted by ultracentrifugation $(186,000 \times g, 40 \mathrm{~min})$ and the soluble fraction was incubated with Streptavidin-linked resin for $10-14 \mathrm{~h}$, rotating at $4{ }^{\circ} \mathrm{C}$. The resin was washed with 10 column volumes of $150 \mathrm{mM} \mathrm{NaCl}, 20 \mathrm{mM}$ Tris- $\mathrm{HCl}$ $\mathrm{pH}$ 8.0, $1 \mathrm{mM}$ BME, $0.1 \%$ DDM, and $0.01 \%$ CHS for hTRPV6, and without CHS for TRPV6 ${ }^{*}$ and TRPV6 ${ }^{*}-\mathrm{Y}_{466 \mathrm{~A}}$. The protein was eluted in the same buffer supplemented with $2.5 \mathrm{mM}$ D-desthiobiotin, and was further purified by size exclusion chromatography. The tetrameric peak fractions were pooled and for hTRPV6-Y467A, the protein was reconstituted in amphipols and concentrated to $0.35-0.45 \mathrm{mg} / \mathrm{ml}$ for cryo-EM sample preparation. For structural studies using crystallography, TRPV6 ${ }^{*}$ and TRPV6*-Y466A were both concentrated to $2.5 \mathrm{mg} / \mathrm{ml}$. For both crystallographic and cryo-EM studies of TRPV6 in complex with 2-APB, $1 \mathrm{mM} 2$-APB was added to every buffer throughout the purification.

Crystallization and Structure Determination. Crystals of purified TRPV6 ${ }^{*}$ were grown in the hanging drop configuration at $20^{\circ} \mathrm{C}$ in the same condition as described previously ${ }^{49}$ : it consists of a reservoir solution containing $20-24 \%$ PEG $350 \mathrm{MME}, 50 \mathrm{mM} \mathrm{NaCl}$, and $50 \mathrm{mM}$ Tris- $\mathrm{HCl} \mathrm{pH} \mathrm{8.0-8.5}$. Prior to crystallization, the protein was subjected to ultracentrifugation (Ti100 rotor, 86,500 $\times g, 40 \mathrm{~min}$, $4^{\circ} \mathrm{C}$ ) to remove any aggregated protein. TRPV $6^{\star}$ purified in the presence of $1 \mathrm{mM}$ 2-APB produced larger crystals compared to those prepared from purified TRPV ${ }^{*}$ incubated with the 2 -APB for $3 \mathrm{~h}$ prior to setting up crystallization trays. The mono-brominated 2-APB (2-APB-Br) was synthesized according to the previously described procedure ${ }^{51}$. Commercially available 1,4-dibromobenzene was monolithiated with ${ }^{n} \mathrm{BuLi}$ and then reacted with commercially available phenylboronic acid pinacol ester to yield borinic acid. Borinic acid was subsequently esterified with 2-aminoethanol to yield the desired 2-APB-Br. A 100-mM stock of 2-APB-Br was prepared in DMSO and added to purified TRPV6* and TRPV6 ${ }^{*}$-Y466A to a final concentration of $1 \mathrm{mM}$. These mixtures were incubated for $3 \mathrm{~h}$ on ice prior to 
setting up crystallization trays. Crystals were cryo-protected by serial transfer into buffers composed of $100 \mathrm{mM} \mathrm{NaCl}, 100 \mathrm{mM}$ Tris-HCl pH 8.2, 0.5 mM DDM, and $50 \mathrm{mM}$ ammonium formate, and containing increasing concentrations of PEG 350 MME, with the maximum concentration of $33-36 \%$, and then flash frozen in liquid nitrogen.

X-ray diffraction data collected at APS (beamlines 24-ID-C/E), NSLSII (beamline 17-ID), or ALS (beamline 5.0.2) were indexed, integrated, and scaled using $\mathrm{XDS}^{52}$ or HKL2000 53 . The initial phase information and structures were solved by molecular replacement using Phaser ${ }^{54}$ and the structure of rat TRPV6* (PDB ID: 5WO7) as a search probe. Most of TRPV6*, including the ankyrin repeat domain, $\mathrm{S} 1-\mathrm{S} 4$, the pore module and the C-terminal hook, were similar to the TRPV6 $6_{\text {cryst }}$; the rest of the structure was built using the omit map as a guide. The robust electron density for the S4-S5 linker was evident from initial phases obtained by molecular replacement and map features improved further during refinement. The model was refined by alternating cycles of building in COOT $^{55}$ and automatic refinement in Phenix $^{56}$ or Refmac ${ }^{57}$.

\section{Reconstitution of hTRPV6-Y467A into Amphipols. hTRPV6-Y467A was} reconstituted into amphipols as described previously ${ }^{41}$. Briefly, $0.48 \mathrm{mg}$ of hTRPV6-Y467A was incubated with amphipols at $4{ }^{\circ} \mathrm{C}$ with constant rotation in a 3:1 amphipols:hTRPV6-Y467A mass ratio. After $3 \mathrm{~h}, 7-10 \mathrm{mg}$ of Bio-beads SM2 prewet in buffer $(20 \mathrm{mM}$ Tris, $\mathrm{pH} 8.0,50 \mathrm{mM} \mathrm{NaCl})$ was added to the protein-amphipols mixture. This mixture was then rotated overnight at $4^{\circ} \mathrm{C}$.

\section{Cryo-EM Sample Preparation and Data Collection. The cryo-EM grids for} hTRPV6-Y467A or hTRPV6-Y467A 2 -APB were prepared as described previously ${ }^{41}$. In short, the gold-coated grids were plasma treated prior to sample application. A Vitrobot Mark IV was used to plunge freeze the grids after the application of $3 \mu \mathrm{l}$ protein solution with $100 \%$ humidity at $5{ }^{\circ} \mathrm{C}$, a blot time of 2 or $3 \mathrm{~s}$, the blot force set to 3 , and a wait time of $20 \mathrm{~s}$. A concentration of $0.35 \mathrm{mg} / \mathrm{ml}$ was used for the amphipols-solubilized protein.

The hTRPV6-Y467A data were collected on a Tecnai F30 Polara $\left(\mathrm{C}_{\mathrm{s}} 2.26 \mathrm{~mm}\right)$, operating at $300 \mathrm{kV}$, equipped with a Gatan K2 Summit electron detection (DED) camera (Gatan, Pleasanton, CA, USA) using Leginon ${ }^{58}$. A total of 3272 micrographs were collected with a pixel size of $0.98 \AA$ across a defocus range of -1.5 to $-3.5 \mu \mathrm{m}$. The total dose, $\sim 67 \mathrm{e}^{-} \AA^{-2}$, was attained by using a dose rate of $\sim 8.0 \mathrm{e}^{-1}$ pixel $^{-1} \mathrm{~s}^{-1}$ across 40 frames for $8 \mathrm{~s}$ total exposure time. The hTRPV6-

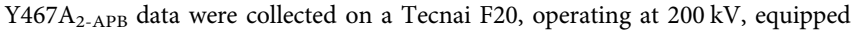
with a Gatan K2 Summit electron detection (DED) camera (Gatan, Pleasanton, CA, USA) using Leginon ${ }^{58}$. For hTRPV6-Y467A 2 -APB, a total of 2112 micrographs were collected with a pixel size of $1.22 \AA$ across a defocus range of -1.5 to $-3.5 \mu \mathrm{m}$. The total dose, $\sim 43 \mathrm{e}^{-1} \AA^{-2}$, was attained by using a dose rate of $\sim 8.0 \mathrm{e}^{-1}$ pixel $^{-1} \mathrm{~s}^{-1}$ across 40 frames for $8 \mathrm{~s}$ total exposure time.

Image Processing. Frame alignment was done using MotionCor $2^{59}$. For both data sets, CTF correction was carried out using Gctf ${ }^{60}$ on nondoseweighted micrographs, while subsequent data processing was done on doseweighted micrographs. The data processing were performed using Relion $2.1^{61}$. For both data sets, between 1000 and 1500 particles were manually selected to generate $2 \mathrm{D}$ classes that were used as templates for automated particle picking. Five 2D classes were used as references for the automated picking of 642,142 particles from the 3272 hTRPV6-Y466A micrographs. The particle images were binned to a pixel size of $1.96 \AA$, and screened using $2 \mathrm{D}$ classification. The remaining particles were subjected to $3 \mathrm{D}$ classification with $\mathrm{C} 1$ symmetry using an hTRPV6 cryo-EM structure (EMDB-7120) low pass filtered to $40 \AA$ as a reference. Two additional rounds of 3D classification were performed on $0.98 \AA$ pixel size particle images with C4 symmetry imposed to generate a more homogenous set of 115,126 particles. To generate the final 3D map, we processed this subset of particles using 3D-auto-refine procedure followed by a postprocessing procedure in Relion 2.1 .

The hTRPV6-Y467A 2 APB data set was processed in a similar workflow to that described above and the reported resolutions were estimated using the FSC $=0.143$ criterion $^{62}$ on masking-effect-corrected FSC curves calculated between two independent half-maps ${ }^{63}$. The local resolutions were estimated with unfiltered half maps using ResMap ${ }^{64}$ and EM density visualization was done in UCSF Chimera ${ }^{65}$.

Fura 2-AM Measurements. The intracellular $\mathrm{Ca}^{2+}$ measurements from HEK 293 cells expressing different TRP channel constructs (see above) were performed similarly to those described previously ${ }^{39,49}$. Briefly, to measure 2-APBdependent inhibition of TRPV6 activity, cells expressing TRPV6 constructs were suspended in $\mathrm{Ca}^{2+}$-free buffer and transferred to a quartz cuvette. After an initial 100 -s equilibration period, $2-\mathrm{APB}$ was added to the cuvette and incubated for another $100 \mathrm{~s}$, before $2 \mathrm{mM} \mathrm{CaCl}_{2}$ was added. For the TRPV6-E402A mutant, $5 \mathrm{mM}$ instead of $2 \mathrm{mM} \mathrm{CaCl}_{2}$ was added. For intracellular $\mathrm{Ca}^{2+}$ measurements from HEK 293 cells expressing TRPV1, TRPV2, or TRPV3, the experiments were carried out with prewarmed modified HBS $(118 \mathrm{mM} \mathrm{NaCl}, 4.8 \mathrm{mM} \mathrm{KCl}, 1 \mathrm{mM}$
$\mathrm{MgCl}_{2}, 2.5 \mathrm{mM} \mathrm{CaCl}, 5 \mathrm{mM}$ D-glucose, $10 \mathrm{mM}$ HEPES pH 7.4). Briefly, HEK 293 cells expressing TRPV $1 / 2 / 3$ channels were centrifuged at $600 \times g$, resuspended in modified HBS containing $5 \mu \mathrm{g} / \mathrm{mL}$ of Fura 2-AM (Life Technologies), and incubated at $37^{\circ} \mathrm{C}$ for $45 \mathrm{~min}$. Next, the cells were centrifuged for $5 \mathrm{~min}$ at $600 \times \mathrm{g}$, resuspended in prewarmed, modified HBS, and incubated again at $37^{\circ} \mathrm{C}$ for $20-30$ min in the dark. The cells were subsequently pelleted and washed twice, and then resuspended in modified HBS for fluorescence measurements. Intracellular $\mathrm{Ca}^{2+}$ was measured by taking the ratio of fluorescence $(510 \mathrm{~nm})$ emitted from Fura 2AM after excitation at either a wavelength of $340 \mathrm{~nm}$ (excites $\mathrm{Ca}^{2+}$ bound Fura 2$\mathrm{AM})$ or $380 \mathrm{~nm}$ (excites $\mathrm{Ca}^{2+}$ free Fura 2-AM). The excitation wavelength was switched at

1-s intervals. $\Delta F_{340} / F_{380}$ was measured as the difference between baseline $F_{340} / F_{380}$ ratio recorded before the addition of $\mathrm{Ca}^{2+}$ (for TRPV6 experiments) or 2-APB (for TRPV1-3) and the maximum $F_{340} / F_{380}$ after addition of either $\mathrm{Ca}^{2+}$ or 2-APB. Data were normalized to $\Delta F_{340} / F_{380}$ in the absence of 2-APB (for TRPV6 constructs) or the $\Delta F_{340} / F_{380}$ value at saturating concentrations of 2-APB (for TRPV1/ 2/3). All Fura2-AM-based fluorescence measurements were conducted using a QuantaMaster ${ }^{\mathrm{TM}} 40$ (Photon Technology International) spectrofluorometer at room temperature in a quartz cuvette under constant stirring.

Data availability. Data supporting the findings of this manuscript are available from the corresponding author upon reasonable request. Crystal structure coordinates have been deposited in the Protein Data Bank (PDB) under Accession nos. 6D7O (TRPV6* ${ }_{2-\mathrm{APB}}$ ), 6D7P (TRPV6*-Y466A), 6D7Q (TRPV6*-Y466A ${ }_{2-\mathrm{APB}}$ ), 6D7V (TRPV6 ${ }_{2-\mathrm{APB}-\mathrm{Br}}$ ), and 6D7X (TRPV6*-Y466A ${ }_{2 \text {-APB-Br }}$ ). Cryo-EM density maps have been deposited in the Electron Microscopy Data Bank (EMDB) under Accession nos. EMD-7824 (hTRPV6-Y467A) and EMD-7825 (hTRPV6-Y467A2$\mathrm{APB})$ and the corresponding structure coordinates in the PDB under Accession nos. 6D7S (hTRPV6-Y467A) and 6D7T (hTRPV6-Y467A2-APB).

Received: 30 March 2018 Accepted: 23 May 2018

Published online: 25 June 2018

\section{References}

1. Venkatachalam, K. \& Montell, C. TRP channels. Annu. Rev. Biochem. 76, 387-417 (2007)

2. Nilius, B. \& Owsianik, G. Transient receptor potential channelopathies. Pflug Arch. Eur. J. Phy 460, 437-450 (2010).

3. Peng, J. B. et al. Human calcium transport protein CaT1. Biochem. Biophys. Res. Commun. 278, 326-332 (2000)

4. Wissenbach, U. et al. Expression of CaT-like, a novel calcium-selective channel, correlates with the malignancy of prostate cancer. J. Biol. Chem. 276, 19461-19468 (2001).

5. Fleet, J. C., Eksir, F., Hance, K. W. \& Wood, R. J. Vitamin D-inducible calcium transport and gene expression in three Caco-2 cell lines. Am. J. Physiol. Gastrointest. Liver Physiol. 283, G618-G625 (2002).

6. Zhuang, L. et al. Calcium-selective ion channel, CaT1, is apically localized in gastrointestinal tract epithelia and is aberrantly expressed in human malignancies. Lab. Invest. 82, 1755-1764 (2002).

7. Bodding, M., Fecher-Trost, C. \& Flockerzi, V. Store-operated Ca2+ current and TRPV6 channels in lymph node prostate cancer cells. J. Biol. Chem. 278, 50872-50879 (2003).

8. Fixemer, T., Wissenbach, U., Flockerzi, V. \& Bonkhoff, H. Expression of the Ca2+-selective cation channel TRPV6 in human prostate cancer: a novel prognostic marker for tumor progression. Oncogene 22, 7858-7861 (2003).

9. Wissenbach, U. et al. TRPV6 and prostate cancer: cancer growth beyond the prostate correlates with increased TRPV6 Ca2+channel expression. Biochem. Biophys. Res. Commun. 322, 1359-1363 (2004).

10. Taparia, S., Fleet, J. C., Peng, J. B., Wang, X. D. \& Wood, R. J. 1,25Dihydroxyvitamin $\mathrm{D}$ and 25-hydroxyvitamin $\mathrm{D}$--mediated regulation of TRPV6 (a putative epithelial calcium channel) mRNA expression in Caco-2 cells. Eur. J. Nutr. 45, 196-204 (2006).

11. Wissenbach, U. \& Niemeyer, B. A. Trpv6. Handb. Exp. Pharmacol. 179, 221-234 (2007).

12. Bolanz, K. A., Hediger, M. A. \& Landowski, C. P. The role of TRPV6 in breast carcinogenesis. Mol. Cancer Ther. 7, 271-279 (2008).

13. Bolanz, K. A., Kovacs, G. G., Landowski, C. P. \& Hediger, M. A. Tamoxifen inhibits TRPV6 activity via estrogen receptor-independent pathways in TRPV6-expressing MCF-7 breast cancer cells. Mol. Cancer Res. 7, 2000-2010 (2009).

14. Semenova, S. B., Vassilieva, I. O., Fomina, A. F., Runov, A. L. \& Negulyaev, Y. A. Endogenous expression of TRPV5 and TRPV6 calcium channels in human leukemia K562 cells. Am. J. Physiol. Cell. Physiol. 296, C1098-C1104 (2009). 
15. Lehen'kyi, V. et al. TRPV6 determines the effect of vitamin D3 on prostate cancer cell growth. PLoS ONE 6, el6856 (2011).

16. Zheng, X. E. et al. Human PXR-mediated induction of intestinal CYP3A4 attenuates 1alpha,25-dihydroxyvitamin $\mathrm{D}$ (3) function in human colon adenocarcinoma LS180 cells. Biochem. Pharmacol. 84, 391-401 (2012).

17. Bowen, C. V. et al. In vivo detection of human TRPV6-rich tumors with anti-cancer peptides derived from soricidin. PLoS ONE 8, e58866 (2013).

18. Fecher-Trost, C., Weissgerber, P. \& Wissenbach, U. TRPV6 channels. Handb. Exp. Pharmacol. 222, 359-384 (2014).

19. Peng, J. B. et al. CaT1 expression correlates with tumor grade in prostate cancer. Biochem. Biophys. Res. Commun. 282, 729-734 (2001).

20. Lehen'kyi, V., Flourakis, M., Skryma, R. \& Prevarskaya, N. TRPV6 channel controls prostate cancer cell proliferation via $\mathrm{Ca}(2+) / \mathrm{NFAT}$-dependent pathways. Oncogene 26, 7380-7385 (2007).

21. Lehen'kyi, V., Raphael, M. \& Prevarskaya, N. The role of the TRPV6 channel in cancer. J. Physiol. 590, 1369-1376 (2012).

22. Schwarz, E. C. et al. TRPV6 potentiates calcium-dependent cell proliferation. Cell Calcium 39, 163-173 (2006).

23. Haverstick, D. M., Heady, T. N., Macdonald, T. L. \& Gray, L. S. Inhibition of human prostate cancer proliferation in vitro and in a mouse model by a compound synthesized to block Ca2+entry. Cancer Res. 60, 1002-1008 (2000).

24. Landowski, C. P., Bolanz, K. A., Suzuki, Y. \& Hediger, M. A. Chemical inhibitors of the calcium entry channel TRPV6. Pharm. Res. 28, 322-330 (2011).

25. Kovacs, G. et al. Inhibition of the human epithelial calcium channel TRPV6 by 2-aminoethoxydiphenyl borate (2-APB). Cell Calcium 52, 468-480 (2012).

26. Nelson, A. M. et al. 2-APB arrests human keratinocyte proliferation and inhibits cutaneous squamous cell carcinoma in vitro. Preprint at bioRxiv https://www.biorxiv.org/content/early/2018/01/23/249821 (2018).

27. Fu, S. et al. First-in-human phase I study of SOR-C13, a TRPV6 calcium channel inhibitor, in patients with advanced solid tumors. Invest. New Drugs 35, 324-333 (2017).

28. Maruyama, T., Kanaji, T., Nakade, S., Kanno, T. \& Mikoshiba, K. 2APB, 2-aminoethoxydiphenyl borate, a membrane-penetrable modulator of Ins (1,4,5)P-3-induced Ca2+release. J. Biochem.-Tokyo 122, 498-505 (1997).

29. $\mathrm{Xu}, \mathrm{X}$. L. et al. 2-Aminoethoxydiphenyl borate potentiates CRAC current by directly dilating the pore of open orai 1. Sci. Rep. 6, 29304 (2016).

30. Zhuo, R. G., et al. Insights into the stimulatory mechanism of 2aminoethoxydiphenyl borate on trek-2 potassium channel. Neuroscience $\mathbf{3 0 0}$ 85-93 (2015)

31. Colton, C. K., Zhu, M. X. 2-Aminoethoxydiphenyl borate as a common activator of TRPV1, TRPV2, and TRPV3 channels Handb. Exp. Pharmacol. 179, 173-187 (2007).

32. $\mathrm{Hu}, \mathrm{H}$. Z. et al. 2-aminoethoxydiphenyl borate is a common activator of TRPV1, TRPV2, and TRPV3. J. Biol. Chem. 279, 35741-35748 (2004).

33. Chung, M. K., Lee, H., Mizuno, A., Suzuki, M. \& Caterina, M. J. 2aminoethoxydiphenyl borate activates and sensitizes the heat-gated ion channel TRPV3. J. Neurosci. 24, 5177-5182 (2004).

34. Hinman, A., Chuang, H. H., Bautista, D. M. \& Julius, D. TRP channel activation by reversible covalent modification. Proc. Natl. Acad. Sci. USA 103, 19564-19568 (2006).

35. Li, M., Jiang, J. \& Yue, L. Functional characterization of homo- and heteromeric channel kinases TRPM6 and TRPM7. J. Gen. Physiol. 127, 525-537 (2006).

36. Togashi, K., Inada, H. \& Tominaga, M. Inhibition of the transient receptor potential cation channel TRPM2 by 2 -aminoethoxydiphenyl borate (2-APB). Br J. Pharmacol. 153, 1324-1330 (2008).

37. Lievremont, J. P., Bird, G. S. \& Putney, J. W. Mechanism of inhibition of TRPC cation channels by 2-aminoethoxydiphenylborane. Mol. Pharmacol. 68 , 758-762 (2005).

38. Chokshi, R., Fruasaha, P. \& Kozak, J. A. 2-Aminoethyl diphenyl borinate (2APB) inhibits TRPM7 channels through an intracellular acidification mechanism. Channels 6, 362-369 (2012).

39. Singh, A. K., Saotome, K. \& Sobolevsky, A. I. Swapping of transmembrane domains in the epithelial calcium channel TRPV6. Sci. Rep. 7, 10669 (2017).

40. Jiang, Y. et al. X-ray structure of a voltage-dependent $\mathrm{K}+$ channel. Nature 423, 33-41 (2003).

41. McGoldrick, L. L. et al. Opening of the human epithelial calcium channel TRPV6. Nature 553, 233-237 (2018).

42. Gao, Y., Cao, E., Julius, D. \& Cheng, Y. TRPV1 structures in nanodiscs reveal mechanisms of ligand and lipid action. Nature 534, 347-351 (2016).

43. Hughes, T. E. T. et al. Structural basis of TRPV5 channel inhibition by econazole revealed by cryo-EM. Nat. Struct. Mol. Biol. 25, 53-60 (2018).
44. Zubcevic, L. et al. Cryo-electron microscopy structure of the TRPV2 ion channel. Nat. Struct. Mol. Biol. 23, 180-186 (2016).

45. Huynh, K. W. et al. Structure of the full-length TRPV2 channel by cryo-EM. Nat. Commun. 7, 11130 (2016).

46. Autzen, H. E. et al. Structure of the human TRPM4 ion channel in a lipid nanodisc. Science 359, 228-232 (2018).

47. Zimova, L. et al. Intracellular cavity of sensor domain controls allosteric gating of TRPA1 channel. Sci. Signal. 11, 8621 (2018).

48. Yin, Y. et al. Structure of the cold- and menthol-sensing ion channel TRPM8. Science 359, 237-241 (2018)

49. Saotome, K., Singh, A. K., Yelshanskaya, M. V. \& Sobolevsky, A. I. Crystal structure of the epithelial calcium channel TRPV6. Nature 534, 506-511 (2016).

50. Goehring, A. et al. Screening and large-scale expression of membrane proteins in mammalian cells for structural studies. Nat. Protoc. 9, 2574-2585 (2014).

51. Hofer, A. et al. Design, synthesis and pharmacological characterization of analogs of 2-aminoethyl diphenylborinate (2-APB), a known store-operated calcium channel blocker, for inhibition of TRPV6-mediated calcium transport. Bioorg. Med. Chem. 21, 3202-3213 (2013).

52. Kabsch, W. Xds. Acta Crystallogr. D Biol. Crystallogr. 66, 125-132 (2010).

53. Otwinowski, Z. \& Minor, W. Processing of X-ray diffraction data collected in oscillation mode. Method Enzymol. 276, 307-326 (1997).

54. McCoy, A. J. Solving structures of protein complexes by molecular replacement with Phaser. Acta Crystallogr. D Biol. Crystallogr. 63, 32-41 (2007).

55. Emsley, P. \& Cowtan, K. Coot: model-building tools for molecular graphics. Acta Crystallogr. D. Biol. Crystallogr. 60, 2126-2132 (2004).

56. Adams, P. D. et al. PHENIX: A comprehensive python-based system for macromolecular structure solution. Acta Crystallogr. D Biol. Crystallogr. 66, 213-221 (2010).

57. Murshudov, G. N. et al. REFMAC5 for the refinement of macromolecular crystal structures. Acta Crystallogr. D Biol. Crystallogr. 67, 355-367 (2011).

58. Suloway, C. et al. Automated molecular microscopy: the new Leginon system J. Struct. Biol. 151, 41-60 (2005).

59. Zheng, S. Q. et al. MotionCor2: Anisotropic correction of beam-induced motion for improved cryo-electron microscopy. Nat. Methods 14, 331-332 (2017).

60. Zhang, K. Gctf: Real-time CTF determination and correction. J. Struct. Biol. 193, 1-12 (2016).

61. Kimanius, D., Forsberg, B. O., Scheres, S. H. W. \& Lindahl, E. Accelerated cryo-EM structure determination with parallelisation using GPUs in RELION-2. eLife 5, 18722 (2016).

62. Scheres, S. H. \& Chen, S. Prevention of overfitting in cryo-EM structure determination. Nat. Methods 9, 853-854 (2012).

63. Chen, S. et al. High-resolution noise substitution to measure overfitting and validate resolution in 3D structure determination by single particle electron cryomicroscopy. Ultramicroscopy 135, 24-35 (2013).

64. Kucukelbir, A., Sigworth, F. J. \& Tagare, H. D. Quantifying the local resolution of cryo-EM density maps. Nat. Methods 11, 63-65 (2014).

65. Pettersen, E. F. et al. UCSF Chimera-a visualization system for exploratory research and analysis. J. Comput. Chem. 25, 1605-1612 (2004).

66. Smart, O. S., Neduvelil, J. G., Wang, X., Wallace, B. A. \& Sansom, M. S. HOLE: a program for the analysis of the pore dimensions of ion channel structural models. J. Mol. Graph. 14, 354-360 (1996).

\section{Acknowledgments}

We thank the personnel at beamlines 24-ID-C/E of APS and 5.0.1/5.0.2 of ALS and 17-ID AMX of NSLSII. This research used resources of the Advanced Photon Source, a U.S. Department of Energy (DOE) Office of Science User Facility operated for the DOE Office of Science by Argonne National Laboratory under Contract no. DE-AC02-06CH11357. We thank H. Kao for computational support and members of the E.C. Greene Lab for assistance with their fluorimeter. We thank R.A. Grassucci for his assistance with the operation of microscopes when collecting data at the Columbia University Medical Center cryo-EM Facility. We also thank S.-X. Deng and A.S. Wasmuth at the Columbia University Organic Chemistry Collaborative Center (OCCC) for chemically synthesizing the brominated derivative of 2-APB. Funding: L.L.M. is supported by the NIH (T32 GM008224). A.I.S. is supported by the NIH (R01 CA206573 and R01 NS083660), the Amgen Young Investigator Award and the Irma T. Hirschl Career Scientist Award.

\section{Author contributions}

A.K.S., K.S., and A.I.S. designed the project. A.K.S. and K.S. developed expression and purification protocols, designed the constructs, prepared the protein samples for cryoEM and produced crystals, collected and analyzed X-ray crystallography data, carried out Fura-2 experiments, built models, analyzed data and wrote the manuscript. A.K.S. and L. L.M. carried out cryo-EM data collection and processing, analyzed data and wrote the 
manuscript. A.I.S. supervised the project, built models, analyzed data, wrote the manuscript and provided funding.

\section{Additional information}

Supplementary Information accompanies this paper at https://doi.org/10.1038/s41467018-04828-y.

Competing interests: The authors declare no competing interests.

Reprints and permission information is available online at http://npg.nature.com/ reprintsandpermissions/

Publisher's note: Springer Nature remains neutral with regard to jurisdictional claims in published maps and institutional affiliations. (c) (i) Open Access This article is licensed under a Creative Commons Attribution 4.0 International License, which permits use, sharing, adaptation, distribution and reproduction in any medium or format, as long as you give appropriate credit to the original author(s) and the source, provide a link to the Creative Commons license, and indicate if changes were made. The images or other third party material in this article are included in the article's Creative Commons license, unless indicated otherwise in a credit line to the material. If material is not included in the article's Creative Commons license and your intended use is not permitted by statutory regulation or exceeds the permitted use, you will need to obtain permission directly from the copyright holder. To view a copy of this license, visit http://creativecommons.org/ licenses/by/4.0/.

(C) The Author(s) 2018 